Mohammad Abdelhamid:

\title{
La transgression discursive en Egypte à travers les inscriptions murales: le graffiti sur les réseaux sociaux, un nouveau champ de contestation.
}

\begin{abstract}
:
L'objectif principal de cette recherche sera d'étudier les inscriptions murales en Egypte après la révolution de 2011. Le tag, le graffiti et l'art de rue n'avaient quasiment aucune visibilité dans le pays avant cette période de fortes instabilités politiques, quand soudainement ils apparurent à tous les coins de rue.

Ma thèse interroge une thématique particulière à savoir la naissance d'un nouveau public, au sens de Dewey c'est-à-dire un public actif bataillant pour sa liberté. Un nouveau public est naît en tant que producteur d'un nouveau champ de protestation dans la rue, laissant une marque pour publiciser son opinion. Puis viennent à la vie d'autres publics qui assistent à l'émergence de ces nouveaux objets et parfois même les postent sur I'internet. La plupart du temps, ce n'est pas l'artiste qui fait la démarche de publier une photographie de graffiti mais un activiste ou un citoyen lambda. Et c'est là que l'étude prend un tournant bien plus intéressant.

En tant que sémioticien, le travail portera sur l'analyse des graffiti sur les nouveaux murs, c'est-à-dire sur l'internet et plus particulièrement Facebook, où le graffiti accède à une plus large visibilité. Les réseaux sociaux octroient un nouveau sens au graffiti qui n'était pas originellement prévu par l'artiste. La nature locale de l'œuvre se voit d'un seul coup transformée en un message global accessible de par le monde.
\end{abstract}

\section{Agenda:}

Dewey et sa définition du public.

Vérification des critères deweyiens.

Nous sommes tous Khaled Saïd.

Sois avec la révolution

Fais nous entendre ta voix

Notre arme contre leur arme.

Le jeu d'échecs

Conclusion

Author:

PhD student Mohammad Abdelhamid:

- Institut de la communication, University of Sorbonne Nouvelle, 13, rue Santeuil 75231 Paris Cedex 05, France

- 淄 + $33-663628735, ه$ mohammadgegen@hotmail.com 
Je souhaite tout d'abord exprimer ma gratitude la plus sincère et mes remerciements les plus profonds aux webmasters de "We are all Khaled Saïd 》, " Graffiti in Egypt » et « Ganzeer » pour leur soutien et leur autorisation au libre usage des images postées sur

leur page.

L'art de rue contemporain s'apparente à une forme unique d'expression. Plusieurs types d'inscription, plusieurs styles et différentes motivations se bousculent afin de s'accaparer le terme de 'graffiti'. Le tag définit l'écriture d'un nom ou plutôt d'un blaze, rapidement et souvent de manière répétitive, d'un seul trait de marqueur. Le graffiti se trouve être une évolution sophistiquée du tag, en en faisant un design artistique, un pochoir à la bombe parmi tant d'autres techniques. Nous devrions noter l'influence prépondérante du mouvement hip-hop qui est apparu aux Etats-Unis d'Amérique (USA) dans les années 1970. Même si l'inscription murale, ou plus généralement en lieu public, a toujours côtoyé notre existence, c'est à partir de cette période que l'art de rue a rencontré un grand succès et le graffiti made in US a grandement influencé le reste du monde. Nous pouvons aisément l'observer dans le cas égyptien au jour d'aujourd'hui.

Le graffiti émerge souvent en période de trouble politique et de situation économique compliquée. Aux USA, ce mode d'expression s'est surtout développé parmi les communautés minoritaires à cause des tensions raciales et des ségrégations subies au quotidien. La motivation première de l'écriture (c'est ainsi que la définissent les pionniers du graffiti) est de marquer un territoire exactement comme le ferait un animal. Cependant, il faut ajouter une dimension politique intrinsèque au message. Prenons un exemple, pour un noir américain le message serait le suivant : comme je n'ai aucun droit dans ce pays je vais m'accorder des droits par la force et de manière illégale, c'est un défi lancé aux autorités. J'annonce : cet espace est mien et j'attends que la police tente de m'attraper.

La visée principale de cette recherche est d'étudier les inscriptions murales en Egypte après la révolution de 2011. Le tag, le graffiti et l'art de rue n'avaient quasiment aucune visibilité dans le pays avant cette période de fortes instabilités politiques, quand soudainement ils apparurent à tous les coins de rue.

Ma thèse interroge une thématique particulière à savoir la naissance d'un nouveau public, au sens de John Dewey ${ }^{1} c^{\prime}$ est-à-dire un public actif bataillant pour sa liberté. Un nouveau public est naît en tant que producteur d'un nouveau champ de protestation dans la rue, laissant une marque pour publiciser son opinion. Puis viennent à la vie d'autres publics qui assistent à l'émergence de ces nouveaux objets et parfois même les postent sur l'internet. La plupart du temps, ce n'est pas l'artiste qui fait la démarche de publier une photographie de graffiti mais un activiste ou un citoyen lambda. Et c'est là que l'étude prend un tournant bien plus intéressant.

En tant que sémioticien, le travail portera sur l'analyse des graffitis sur les nouveaux murs, c'est-à-dire sur l'internet et plus particulièrement Facebook, où le graffiti accède à une plus large visibilité. Les réseaux sociaux octroient un nouveau sens au graffiti qui n'était pas originellement prévu par l'artiste. La nature locale de l'œuvre se voit d'un seul coup transformée en un message global accessible de par le monde. Au début de la révolution, les médias égyptiens n'accordaient aucune attention à ce qui se passait dans le pays, donc la publication de telles images sur l'internet était essentiellement destinée aux égyptiens vivant hors des frontières nationales et aux médias étrangers. Il s'agit par conséquent d'étudier le transfert d'un mur urbain à un mur numérique et virtuel, d'un espace à une place dans laquelle il y a beaucoup plus de circulation de l'information comme le proclamait Michel De Certeau².

La triple transgression ayant lieu durant le passage de l'image d'un mur urbain à un mur digital nous mène à un point capital de notre étude. Avant tout, l'artiste prend un risque non négligeable en peignant dans un espace public, en second lieu une personne quelconque décide de prendre en photo l'œuvre et de la poster sur

1 DEWEY John, Le public et ses problèmes, Farrago/Editions Léo Scheer, Publications de I'Université de Pau, 2003.

2 DE CERTEAU Michel, L'invention du quotidien, Gallimard, Paris, 1994. 
un réseau social et enfin un surfeur prend son courage à deux mains et va visiter la page en question et va peut-être même, risque ultime face aux renseignements généraux, commenter l'image de la dite œuvre.

Le problème majeur que nous rencontrons concernant une analyse sémiotique, c'est la perte de source du graffiti au moment de son poste sur l'internet. Presque à tous les coups, nous ne disposons d'aucune information au sujet du lieu où se trouve le graffiti, ni le moment et encore moins qui a sévi pour nous faire part d'une telle œuvre. Ceci dit, nous pouvons prendre ces objets transgressifs comme étant une manière anonyme de s'exprimer (ça n'est pas toujours le cas) ; souvent le nom est à cacher pour fuir aux yeux du régime en place.

Pour ce qui est de l'article, le fondement de notre travail se basera sur l'analyse de cinq graffitis provenant principalement de pages, ayant pour thème la révolution égyptienne avant tout, les plus visitées et les plus commentées sur Facebook, tel que « We are all Khaled Saïd » (2 178321 like it · 591433 discuss about it).

\section{Dewey et sa définition du public.}

Sept points peuvent être sélectionnés pour rendre compte de cette notion de public dans la pensée praxéologique de Dewey :

a) Pas de censure dans la discussion ou dans le débat

b) Exiger des enquêtes, c'est la mission du public

c) Tout est basé sur l'expérience

d) Un public « dispersé », « chaotique » et « éclipsé » doit être regroupé

e) Des conséquences d'intérêt public

f) Dernière étape en vu d'une constitution d'un public, l'apparition d'un Nous faisant face à un Eux

g) La reconstruction du public doit être permanente et continue

\section{Vérification des critères deweyiens.}

Une fois la notion de public bien déterminée, nous voilà engagés dans une analyse empirique afin de vérifier si la vision de Dewey peut s'appliquer au cas égyptien. 


\section{Nous sommes tous Khaled Saïd}

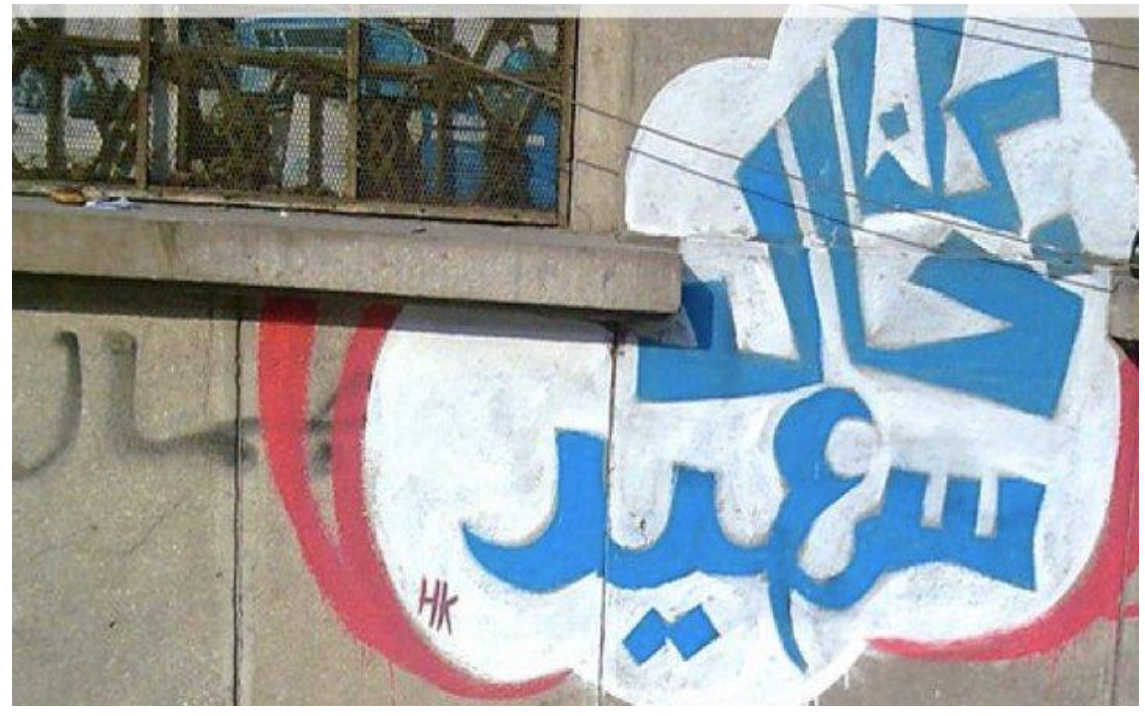

Khaled Saïd fut la goutte d'eau qui a fait déborder le vase. L'histoire de ce jeune alexandrin est considérée par tant d'observateurs comme étant le déclencheur de la révolution. Il avait publié une vidéo sur Youtube révélant le comportement mafieux de policiers se partageant les fruits d'une saisie douanière. Seulement, la saisie est composée d'argent, de tabac mais aussi de drogues. La réponse ne se fait pas attendre, le six juin 2010, il est battu à mort par deux indicateurs de police.

Le message est ici on ne peut plus clair, « Nous sommes tous Khaled Saïd », à proprement parler nous sommes tous des victimes du régime qui a une facilité déconcertante pour ce qui est de la condamnation des citoyens ou plutôt de ses sujets. Sans procès, la sentence tombe, et avant même que quiconque exige une explication, une excuse est fournie. Dans le cas de Khaled, la justification au lendemain ne surprend guère. Khaled était, selon la police, un drogué qui a refusé de coopérer avec les forces de l'ordre et c'est pour cette raison que l'intervention s'est très mal déroulée. Le fautif est facilement désigné.

Ce qui importe dans notre analyse, c'est l'usage d'un « Nous ». Le passant se trouve, sans son accord, affilié à un collectif, un « Nous » qui s'oppose à un « Eux », à savoir le régime. Soulignons ici la réflexivité de la phrase. Les membres de ce groupe sont donc conscients de faire partie d'une communauté. Par coutume, c'est là la première définition donnée à un public ; c'est-à-dire prendre conscience de son appartenance à un collectif. Le « tous » est synonyme d'une expérience collective, nous somme « TOUS » victimes de ce régime exactement comme Khaled ou du moins nous connaissons tous, dans notre entourage, une personne qui a souffert d'humiliation de la part de représentants du régime.

Concernant la peinture en tant que telle, le style est assez occidental avec des formes rigides, carrées et angulaires. Le graffeur affilie le mouvement révolutionnaire à un monde occidental qui serait connu pour sa culture de la démocratie et pour son goût pour les libertés. Il opte pour un mode d'expression occidental dans le but de transmettre un message égyptien. Tous les égyptiens sont Khaled Saïd mais de manière occidentale.

Nous voyons également que le dessin prend la forme d'un nuage. Avec des couleurs claires et vives, le graff semble monter droit vers le ciel. Illusion de bandes dessinées mais le nuage souligne, non seulement le côté enfantin mais surtout, un aspect angélique du message qui est à la fois chargé d'une foi puissante. La personne est décédée et se trouve déjà dans l'au-delà alors l'artiste lui envoie un message de soutien pour lui prouver qu'il n'est pas mort pour rien. Nous nous inspirons de son expérience, il sera le champion de la cause égyptienne. 


\section{Sois avec la révolution}

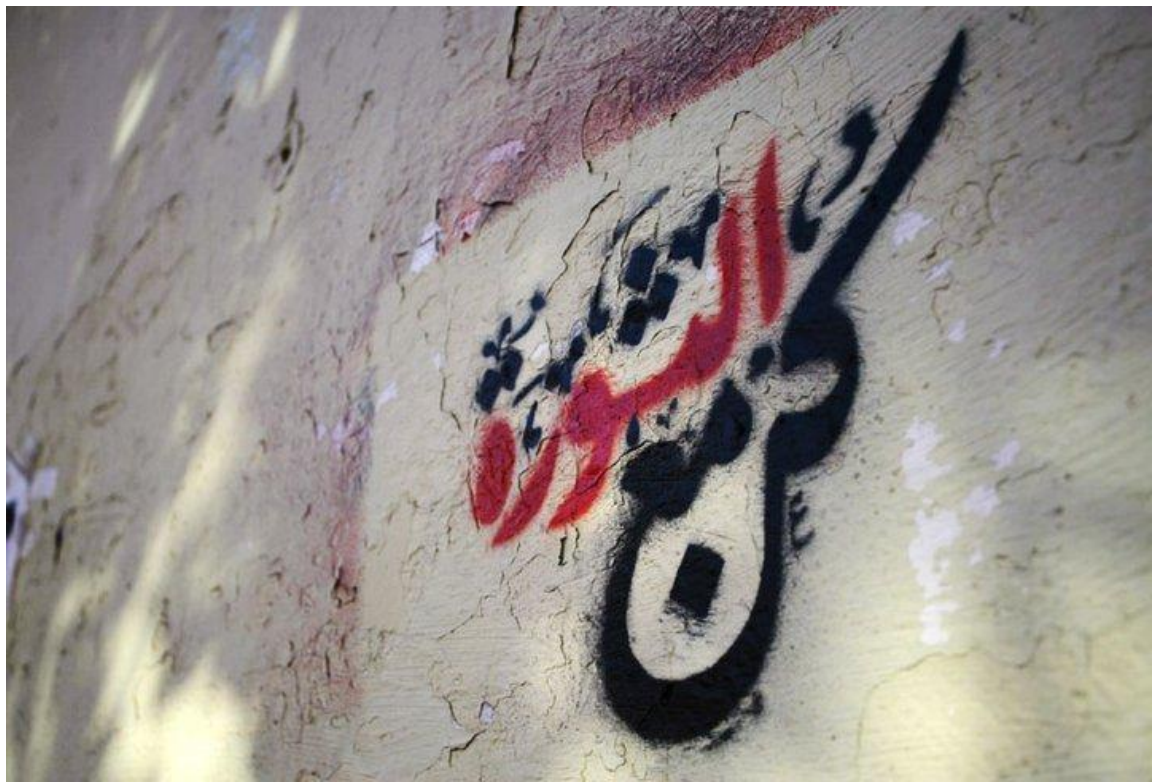

Ce qui ressort tout de suite de ce graff, ce sont les couleurs du drapeau égyptien : noir, blanc et rouge. Pochoir effectué à la bombe, le style est complexe et précis. Contrairement au graff précédent, celui-ci apporte une touche orientale. La calligraphie est employée dans ce cas, I'artiste utilise un mode d'expression a priori occidental mais se l'approprie et le transpose à une situation local. Quoi de mieux que d'opter pour la calligraphie pour effectuer un graffiti ? cet art oriental offre une liberté totale à l'artiste, il peut ainsi écrire, les mots ou même les lettres, dans l'ordre qu'il souhaite. Nous pouvons donc surnommer ce type de travail le « calligraffiti ».

En effet, le graffiti et la calligraphie se rencontrent et vont se balader main dans la main dans les rues du Caire. « Etre » et « avec » sont liés alors qu'ils ne sont pas supposés l'être. Puis « révolution » nous saute aux yeux grâce à sa couleur typique, le rouge, rouge sanguin. La révolution est imprégnée de sang !

Et soudainement, quelque chose attire notre attention. La ponctuation de « révolution » se distingue, elle n'est plus rouge mais noire comme le reste du pochoir. Elle s'ajoute à l'entité « Etre avec », ils composent à eux deux le même CORPS. A traduire donc comme suit : la révolution est le sang qui coule dans nos veines.

Alors, nous pouvons interpréter cela de la manière suivante : nous ne sommes pas censés être ave la révolution, ni aller vers elle pour la rendre effective. Non ! cette révolution est incorporée en chacun de nous, elle est en nous. La révolution est dans notre nature. Nous ne pouvons la combattre, tout comme nous ne pouvons faire face à notre nature.

Nous nous trouvons donc face à une double contrainte, la première provenant de l'auteur, grâce à son injonction à travers l'impératif du verbe, et la seconde nous serait supposément imposée par notre nature. Nous devons être avec la révolution, nous n'y sommes pas conviés, nous devons être en plein dedans.

Une autre interprétation pourrait exprimer une version moins directe. « Etre » et « avec » représenteraient le citoyen qui aurait donc en son pouvoir de ponctuer la révolution comme bon lui semble grâce à sa participation. Tout dépendrait de lui ! 


\section{Fais nous entendre ta voix}

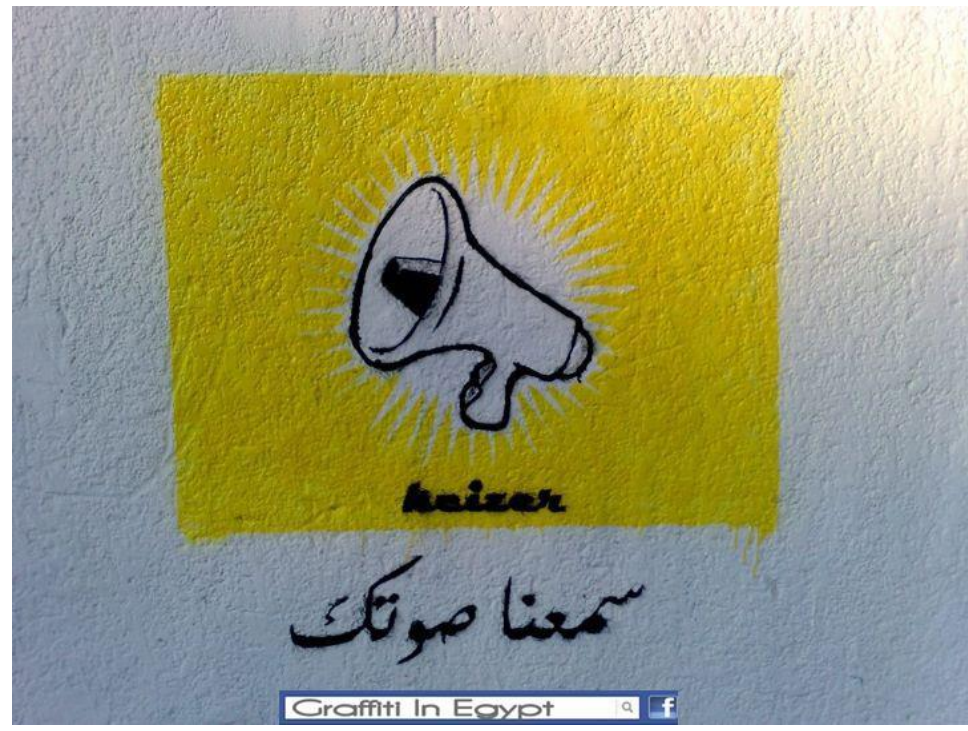

Etre un soutien de la révolution ne signifie guère rester chez soi et être convaincu du bien-fondé de celle-ci, et qu'elle est le chemin à suivre pour la nation. Ca ne suffit pas ! Chaque citoyen doit participer. Encore une fois le destinataire est clairement pointé du doigt. Le « tu » est directement nommé. Toi, oui toi, écoute-moi et fais-nous écouter ta voix. Voici en substance le message dans le texte. Mais ce n'est pas tout, prenez un hautparleur, allez place Tahrir et criez, dites ce qui est enfermé dans votre cœur depuis des années, voire des décennies. Enfin, vous pouvez exprimer votre colère, et même faire part de votre opinion, alors faites-le.

Cette peinture a toutefois quelque chose de particulier. Le jaune n'est pas une couleur que le graffeur égyptien Keizer utilise souvent. Ses habitudes le renvoient plutôt vers le rouge et le noir, ce qui donne à ce graff un sens particulier. Le haut-parleur brille dans une atmosphère ensoleillée. De ce fait, exprimer son opinion en public serait assimilé à un acte de résistance qui nous mènerait depuis les ténèbres à la lumière. Vous ne devez pas vous contenter d'une résistance virtuelle et de transgression sur les réseaux sociaux mais vous devez descendre dans la rue et hurlez votre douleur devant tout le monde. Rendez votre souffrance publique pour enfin voir le bout du tunnel.

\section{Notre arme contre leur arme.}

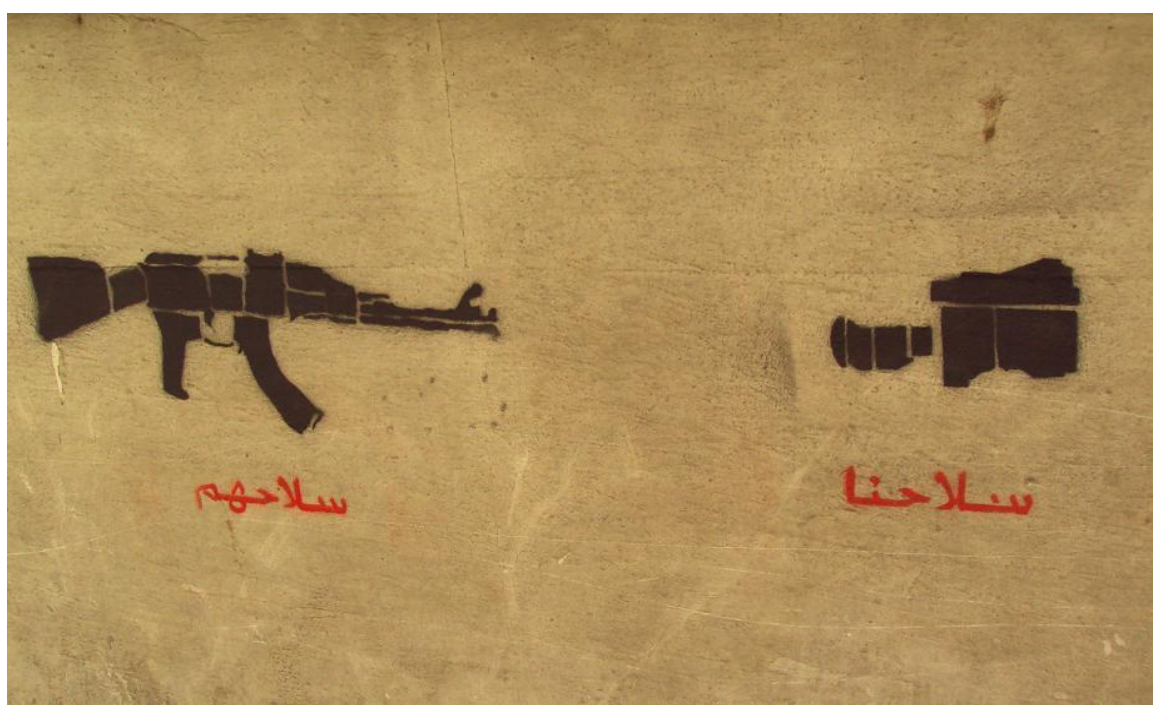


Quand il s'agira d'arme, il faudra combattre l'ennemi avec ses propres ressources. Le révolutionnaire doit prendre les armes s'il y est forcé, mais uniquement des armes pacifiques. Quel antagonisme ! « Notre » arme n'est ni plus ni moins qu'une caméra, donc l'optique des révolutionnaires serait de publier la violence de l'adversaire. La caméra prend le rôle ici du représentant de toutes les nouvelles technologies qui ont servi à combattre le régime, comme les téléphones portables et les réseaux sociaux entre autres. Le militant se doit donc de montrer aux yeux du monde à quel point l'ennemi est brutal. Une klashinkov s'engage dans un face à face avec une caméra. Les deux objets sont dessinés dans un même style, d'une seule et même couleur et se trouvent à la même hauteur. De prime abord, ils paraissent égaux pourtant quand nous y regardons de plus près un sentiment de combat inéquitable nous saisit. Deux opposants se lancent dans un combat mais avec des défenses tout à fait inéquitables. Une caméra est confrontée à une arme de guerre. Une victime faible doit donc batailler avec un « méchant » bourreau tout puissant, c'est-à-dire la police ou même l'armée.

Par conséquent, la population est victime d'une agression atroce mais elle doit résister et révéler au monde la brutalité du régime qui l'opprime au quotidien, de sorte que le reste du monde prendra le parti des citoyens égyptiens. La mission de la population est donc de rendre public les injustices subies constamment. Des citoyens mourront, non pas les armes à la main, mais ils s'en iront pour une noble cause, qui est de publiciser les humiliations du quotidien et de les faire connaître de par le monde. Aux yeux du graffeur, le public a les traits d'un héros. Il est brave, courageux et est prêt à se sacrifier. Il peut donner sa vie dans le but de recouvrir sa liberté.

L'image recèle de bon nombre d'éléments à analyser, le plus capital concerne le destinataire. En tant que lecteur arabe ou égyptien, le passant ou le webnaute n'a pas le choix entre le « Nous » et le «Eux ». La peinture est tellement manichéenne qu'il vaut mieux pencher pour le « bon » côté. Qui plus est, l'arabe se lit de droite à gauche, le destinataire voit donc tout d'abord la caméra avec le « Nous » inscrit en-dessous, qui lui est alors assigné de suite. Les rôles sont partagés sans demander l'avis du passant. Ensuite, ce qui compte plus que tout dans cette image, c'est la place occupée par chaque arme. Se trouver à droite ou à gauche n'est en rien anodin. Dans le monde arabe, autant que dans la tradition judéo-chrétienne, la droite représente le « bien », les anges, tandis que la gauche (la senestre) fait figure de « mal » et incarne la diable.

C'est aussi simple que cela, lorsqu'un message consomme une antithèse manichéenne, la charge symbolique s'en trouve renforcée ${ }^{3}$. Pour la rétention de l'information, il vaut mieux émettre un message simple et clair comportant un bon et un mauvais parti, un noir et un blanc, pas de place pour le gris dans notre monde où la dictature de l'image fait rage. 


\section{Le jeu d'échecs}

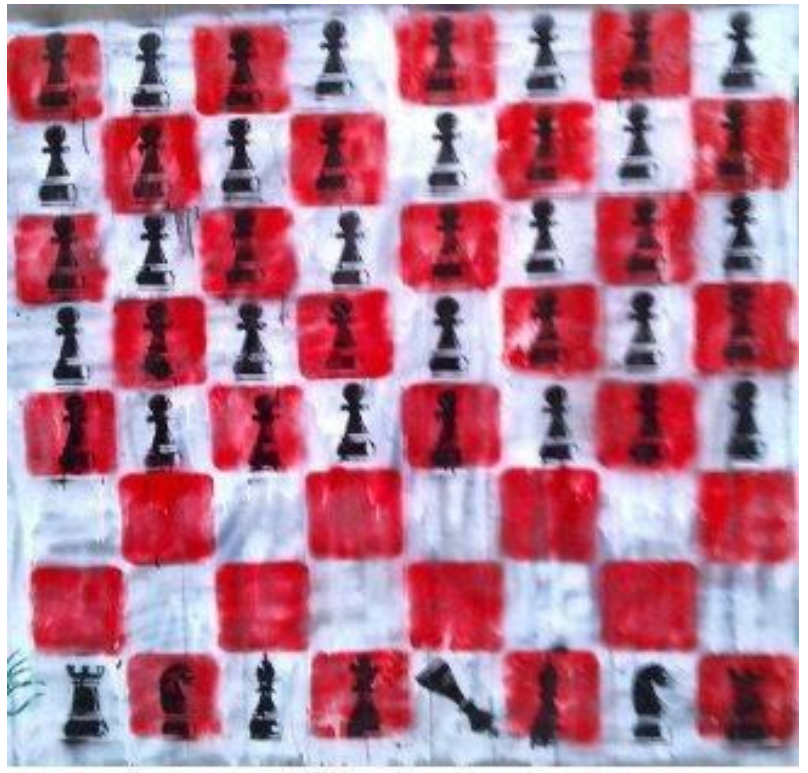

Encore une fois, les références au drapeau égyptien et à l'opposition entre deux camps sont présentes.

Le choix des échecs est très pertinent. Les échecs sont bien évidemment un jeu de stratégie dans lequel prennent place une guerre des nerfs et une guerre d'usure. Le joueur le plus intelligent l'emportera, il n'est pas question de vaincre grâce à ses muscles ou par la force. Un évident message d'espoir est lancé à la population.

Regardons plus attentivement le graff, quarante pions se situent dans la partie haute de l'échiquier alors que huit autres pièces occupent le bas. Mais ces huit pions ne sont pas quelconques, il s'agit là des pièces maîtresses du jeu, le roi, la reine, la tour, le cavalier et ainsi de suite. Cela dit, quelque chose perturbe notre lecture, le roi est retourné, il est battu. Moubarak est donc vaincu, son règne touche à sa fin. Echec et mat ou plutôt « al shaikh mat » en arabe qui se traduit tout simplement par « le roi est mort », ce qui donna naissance à la formule française ou encore à l'anglaise. Mais les autres membres du régime sont toujours sur leurs pieds et tiennent tête aux pions. Le roi est mort, vive le Roi ! Moubarak a lâché prise mais le régime tient toujours et ce avec fierté, il attend le défi lancé par les citoyens sans crainte. Les dirigeants du pays n'ont en rien perdu de leur superbe.

Pourtant, les pions du dessus (ou les citoyens) possèdent un avantage, qui est celui du nombre. Mais pas seulement, ils se retrouvent, comme nous l'avons souligné auparavant, en haut de l'échiquier, ce qui nous donne cette impression qu'ils vont crouler sur les pièces du dessous et les avaler d'un mouvement sans aucune pitié. En fait, le message émis n'est qu'un encouragement ou même une incitation poussant la population à être active au cours de cette révolution. Effectivement, les pions ont besoin d'une rangée de plus pour s'assurer la victoire finale. Donc symboliquement l'idée transcrite dans ce graff est la suivante : un petit effort supplémentaire et la révolution atteindra ses objectifs initiaux.

\section{Conclusion}

En résumé presque tous les éléments sont réunis :

Nous sommes tous des victimes (Expérience (c) et des conséquences d'intérêt public (e)) 
Donc rejoignez la révolution (regrouper les citoyens (d))

Exprimer vos opinions (Pas de censure (a))

Combattez et révélez les injustices (exiger des enquêtes (b) et apparition d'un « Nous »(f))

\section{Et la victoire en sera le résultat}

Un public, au sens de Dewey, a émergé durant la révolution égyptienne. Toutes les conditions, pour la naissance d'un public, sont réunies mise à part l'élément $(\mathrm{g})$ qui manque à notre analyse pour l'instant. Nous avons souligné que le public doit être remis en question en permanence et dans le cas égyptien, il est bien trop tôt pour apporter, ne serait-ce que, des bribes de réponse. Nous ne pouvons pour le moment affirmer que les critères de Dewey sont tous regroupés dans le cas du graffiti, et plus particulièrement en Egypte.

Ajoutons à cela que l'approche de Dewey ne manque d'engendrer quelques questionnements comme par exemple ce « Nous » qui doit émerger pour l'aboutissement d'un public. Le pronom « Nous » peut mener aux pires horreurs dont l'Histoire fut témoin à tous les instants, c'est par ailleurs ce que soutiennent Daniel Dayan et Annabelle Sreberny ${ }^{4}$.

En ce qui concerne les analyses ci-dessus, il y a une évidente tentative de constitution d'un public mais il est compliqué de se prononcer sur la réussite de cet essai pour l'instant. Nous devons certainement patienter quelques années encore et suivre ce qu'il en sera de l'évolution de la situation égyptienne. Le graffiti va-t-il continuer son bonhomme de chemin et inciter les citoyens à se lever à chaque fois qu'un de leur droit sera bafoué ?

D'autres pistes s'ouvrent et seront certainement poursuivies par la suite. Le passage de murs urbains à des murs virtuels a joué un rôle majeur dans la révolution puisque procéder à ce transfert a permis la publicisation en Egypte mais aussi hors des frontières du pays. Au sein même de l'Egypte, l'objectif était clair, regrouper des manifestants ; à l'étranger, le but était tout autre : se mettre l'opinion internationale (si toutefois il y en a bien une) de son côté et constituer un public plus large qui saura défendre celui déjà existant en Egypte. Le combat gagnait donc en puissance dans les médias et surtout sur l'internet.

Le grand souci de cet exposé est que la narration présentée est totalement fictive dans sa constitution chronologique. J'ai moi-même composé un ordre entre ces différents graffitis sans chronologie cohérente. J'ai procédé de la sorte pour faire parler les murs. Très souvent, l'histoire que nous racontent les graffitis est similaire à celle que je soumets ici. Je suis bien conscient que ce travail est loin, très loin d'être abouti mais cela constitue seulement un galop d'essai pour étudier un mode d'expression universel, mais en même temps marginal.

\section{Online references}

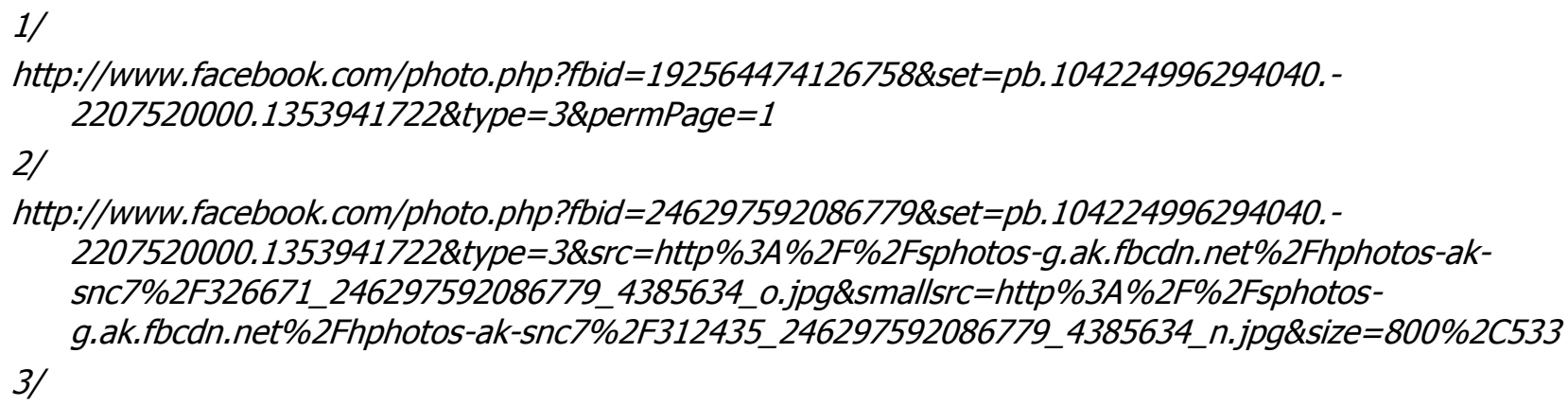

4 DAYAN Daniel (dir.), La terreur spectacle, terrorisme et télévision, De Boeck, Institut national de l'audiovisuel, Bruxelles, 2006. 
http://www.facebook.com/photo.php?fbid=232749430130955\&set=pb.197276033678295. 2207520000.1353929544\&type=3\&theater

4)

http://ganzeer.com/cairostreetart/index.htm/

5/

http://www.facebook.com/photo.php?fbid=186252084757997\&set=pb.104224996294040. 2207520000.1353941722\&type=3\&permPage $=1$

\section{References}

BEN NEFISSA Sarah et DESTREMAU Blandine (dir.), Protestations sociales, révolutions civiles. Transformations du politique dans la Méditerranée arabe, Armand Colin, coll. "Revue Tiers Monde », 2011.

DAYAN Daniel (dir.), La terreur spectacle, terrorisme et télévision, De Boeck, Institut national de l'audiovisuel, Bruxelles, 2006.

DEWEY John, Le public et ses problèmes, Farrago/Editions Léo Scheer, Publications de I'Université de Pau, 2003.

DE CERTEAU Michel, L'invention du quotidien, Gallimard, Paris, 1994.

GANZ Nicholas, Graffiti world: street art from five continents, Abrams, New York, 2006.

GUIBAL Claude, SALAÜN Tangi, L'Egypte de Tahrir, Anatomie d'une révolution, Seuil, Paris, 2011.

LEFEBVRE Henri, La production de l'espace, Editions Anthropos, 1974, Paris.

LEWISOHN Cedar, Street Art, the graffiti revolution, Abrams, New York, 2008.

MAINGUENEAU Dominique, Analyser les textes de communication, Armand Colin, coll. " Lettres sup », Paris, 2007.

OSMAN Tarek, Révolutions égyptiennes, de Nasser à la chute de Moubarak, Les belles lettres, coll. « Le bruit du monde », Paris, 2011.

ZOGHBI Pascal et Don Karl, Le Graffiti arabe, Eyrolles, Paris, 2012. 